\title{
Serum proinsulin levels are disproportionately increased in elderly prediabetic subjects
}

\author{
L.Mykkänen $^{1,2}$, S.M.Haffner ${ }^{2}$, J. Kuusisto ${ }^{1}$, K. Pyörälä ${ }^{1}$, C.N.Hales ${ }^{3}$, M. Laakso ${ }^{1}$ \\ ${ }_{1}^{1}$ Department of Medicine, University of Kuopio, Kuopio, Finland \\ ${ }^{2}$ Division of Clinical Epidemiology, Department of Medicine, University of Texas Health Science Center, San Antonio, Texas, \\ USA \\ ${ }^{3}$ Department of Clinical Biochemistry, Addenbrooke's Hospital, Cambridge, UK
}

\begin{abstract}
Summary Insulin resistance and impaired insulin secretion are thought to be the primary defects in the pathogenesis of non-insulin-dependent diabetes mellitus (NIDDM). Disproportionately increased proinsulin relative to insulin levels are suggested to be an early indicator of a failing pancreas. We examined the relationship of fasting specific insulin, proinsulin, and 32, 33 split proinsulin concentrations, and the proinsulin: insulin ratio to the risk of developing NIDDM 3.5 years later in 65-74-year-old non-diabetic Finnish subjects participating in a populationbased study ( $n=892$ ) on diabetes and heart disease. Altogether 69 subjects developed NIDDM over a 3.5-year follow-up (cases). The cases were compared to randomly-selected gender-matched control subjects $(n-69)$ and control subjects matched for gender, glucose tolerance status (normal or impaired), and body mass index $(n=69)$. There were no differences in insulin concentrations between cases and random or matched control subjects [median and interquartile range; 123 (77-154), 108 (74-143), 118 (83-145) $\mathrm{pmol} / \mathrm{l}, p=0.271]$. Random control subjects had lower proinsulin and 32,33 split proinsulin con-
\end{abstract}

centrations and split proinsulin: insulin ratios compared to cases [5.7 (3.8-9.0) vs $7.3(4.8-10.0) \mathrm{pmol} / 1$, $p=0.005 ; 7.3(4.5-13.0)$ vs $10.4(7.1-18.0) \mathrm{pmol} / 1$, $p=0.002 ; 0.073(0.057-0.110)$ vs $0.097(0.060-0.135)$, $p=0.003]$. Matched control subjects had lower proinsulin concentrations and proinsulin: insulin ratios compared to cases $[5.9(4.0-7.7)$ vs 7.3 (4.8$10.0) \mathrm{pmol} / \mathrm{l}, p=0.019 ; 0.048(0.035-0.071)$ vs 0.064 $(0.045-0.100), p=0.008]$. When cases were compared to matched control subjects a 1 SD increase in baseline proinsulin: insulin ratio was associated with a 1.37-fold risk $(p=0.020)$ of developing diabetes. Moreover, this association was independent of fasting glucose concentration at baseline. Thus, in elderly prediabetic subjects disproportionately increased proinsulin concentration, an indicator of defective insulin secretion, is associated with conversion to diabetes over a short time period. [Diabetologia (1995) 38: 1176-1182]

Key words Proinsulin, insulin, insulin secretion, noninsulin-dependent diabetes mellitus, epidemiology, follow-up study.
Insulin resistance [1-8] and impaired insulin secretion [9-11] are thought to be the primary defects in the pathogenesis of non-insulin-dependent diabetes mel-

Received: 6 July 1994 and in revised form: 4 February 1995

Corresponding author: Dr. L. Mykkänen, Department of Medicine, University of Kuopio, P. O. Box 1777, FIN-70210 Kuopio, Finland

Abbreviations: NIDDM, Non-insulin-dependent diabetes mellitus; IRI, immunoreactive insulin; OR, odds ratio; CI, confidence interval. litus (NIDDM). Low acute insulin response to intravenous glucose was not associated with the risk of NIDDM [6] or was only a minor risk factor compared to insulin resistance [8] in the two prospective studies which have assessed both insulin resistance and acute insulin response in subjects who subsequently developed NIDDM. It is, however, not known whether acute insulin response is the optimal measure of insulin secretion. Impaired beta-cell function is manifested in part by disproportionately increased proinsulin relative to insulin levels [12]. Subjects with NIDDM have a disproportionate elevation 
Table 1. Baseline characteristics of the study population

\begin{tabular}{|c|c|c|c|c|c|c|}
\hline & \multicolumn{3}{|l|}{ Subjects } & \multicolumn{3}{|l|}{$p$-values } \\
\hline & $\begin{array}{l}\text { NIDDM } \\
\text { cases } \\
(n=69)\end{array}$ & $\begin{array}{l}\text { Random } \\
\text { control } \\
(n=69)\end{array}$ & $\begin{array}{l}\text { Matched } \\
\text { control } \\
(n=69)\end{array}$ & Overall & $\begin{array}{l}\text { Cases vs } \\
\text { random } \\
\text { control subjects }\end{array}$ & $\begin{array}{l}\text { Cases vs } \\
\text { matched } \\
\text { control subjects }\end{array}$ \\
\hline Male/female & $30 / 39$ & $30 / 39$ & $30 / 39$ & $\mathrm{~m}$ & $\mathrm{~m}$ & $\mathrm{~m}$ \\
\hline Age (years) & $68.6 \pm 2.7$ & $69.0 \pm 2.7$ & $68.9 \pm 3.0$ & 0.721 & 0.435 & 0.568 \\
\hline Proportion with IGT (\%) & 69.6 & $17.4^{\mathrm{b}}$ & 69.6 & $<0.001$ & $<0.001$ & $\mathrm{~m}$ \\
\hline Fasting plasma glucose $(\mathrm{mmol} / \mathrm{l})$ & $6.2 \pm 0.7$ & $5.8 \pm 0.5^{\mathrm{b}}$ & $6.0 \pm 0.7^{\mathrm{a}}$ & $<0.001$ & $<0.001$ & 0.016 \\
\hline $2-\mathrm{h}$ plasma glucose $(\mathrm{mmol} / \mathrm{l})$ & $8.4 \pm 1.7$ & $6.3 \pm 1.7^{b}$ & $8.0 \pm 1.7$ & $<0.001$ & $<0.001$ & 0.093 \\
\hline
\end{tabular}

Random control subjects matched for gender only

Matched control subjects matched for gender, glucose tolerance status (normal or impaired glucose tolerance) and BMI $p$-value for ANOVA or chi-squared test $\mathrm{m}$, matching criteria; IGT, impaired glucose tolerance.

${ }^{\mathrm{a}} p<0.05,{ }^{\mathrm{b}} p<0.001$

Data are mean \pm SD of proinsulin concentrations [13-17]. According to cross-sectional studies, absolute proinsulin concentrations are also elevated in subjects with impaired glucose tolerance [15, 18-22]. Although some studies found proinsulin levels to be accompanied by similarly elevated insulin levels [15], in several studies subjects with impaired glucose tolerance had elevated fasting proinsulin relative to insulin concentrations $[19,21,22]$. Few studies have investigated prospectively whether proinsulin concentration predicts conversion to NIDDM. Therefore, we examined insulin, intact proinsulin, and 32,33 split proinsulin concentrations and proinsulin : insulin and split proinsulin: insulin ratios, and their relationship to the risk of developing diabetes 3.5 years later in 65-74-year-old non-diabetic Finnish subjects participating in a population-based study on diabetes and heart disease.

\section{Subjects and methods}

Subjects. The baseline cross-sectional study was conducted in Kuopio, eastern Finland from 1986 to 1988 . The composition [23] and representativeness [24] of the study population which was a random sample of the Kuopio population aged 6574 years have been previously described in detail. Altogether, 1,054 subjects participated $(88 \%)$ in the follow-up study performed from 1990 to 1991, on average 3.5 years (42 4 months) after the baseline cross-sectional study as reported previously [25]. A total of 159 subjects who had diabetes at baseline were excluded from the study due to the presence of NIDDM. The 69 subjects who developed NIDDM during the 3.5-year follow-up are termed "cases" in this case-control study. Two groups of control subjects were selected from the participants who remained non-diabetic during the follow-up: 1) random control subjects $(n=69)$ who were matched for gender only and 2) matched control subjects $(n=69)$ who were matched for gender, glucose tolerance status (normal or impaired glucose tolerance) and body mass index (BMI). Thus, this is a case-control study nested within a prospective cohort study. Unlike the procedure in a normal case-control study, assessment of the risk factors was made before the development of NIDDM.
Methods. Body mass index (BMI) was calculated as weight $(\mathrm{kg})$ divided by height $(\mathrm{m})$ squared and was used as an index of overall adiposity. The waist-hip ratio was used as a measure of body fat distribution as previously described [25]. Previously diagnosed diabetes was considered to be present, if the diagnosis had been made earlier by a physician [23]. The World Health Organization plasma glucose criteria for diabetes were used in the classification of subjects without previously diagnosed diabetes [26].

Laboratory methods. Blood samples were taken between 07.30 and 09.30 hours after a $12-\mathrm{h}$ fast. All subjects underwent a $75-\mathrm{g}$ oral glucose tolerance test both in the baseline cross-sectional study and at the 3.5-year follow-up. Venous blood samples for glucose concentration were taken before and $2 \mathrm{~h}$ after the glucose load. Plasma glucose was determined by the glucose oxidase method (Glucose \& Stat HGA-1120 analyzer; Daiichi, Kyoto, Japan). Fasting immunoreactive insulin (IRI) was determined by a double-antibody solid-phase radioimmunoassay [27] (Phadeseph Insulin RIA 100; Pharmacia Diagnostics $\mathrm{AB}$, Uppsala, Sweden) which also detects proinsulin and proinsulin conversion products with considerable sensitivity. The interassay coefficient of variation for IRI was less than $8.7 \%$.

Fasting serum specific insulin, intact proinsulin, and 32, 33 split proinsulin were determined from samples stored at $70^{\circ} \mathrm{C}$ for an average of 6.4 years (67-92 months) by means of highly specific two-site monoclonal-antibody-based immunoradiometric assays with interassay coefficients of variation of less than $10 \%$ [28]. Data on stability of insulin-like molecules under these storage conditions for over 6 years are not available. However, the groups did not differ for the length of storage (mean $\pm \mathrm{SD}, 76.6 \pm 7.5,75.9 \pm 6.0$ and $77.0 \pm 7.0$ month in diabetic cases, random control subjects and matched control subjects, respectively; $p=0.624$ ) and it seems highly unlikely that there were differences in stability during storage between the groups.

In the specific insulin assay, the cross-reactivity with both intact proinsulin and 32, 33 split proinsulin was $5 \%$. There was no detectable cross-reactivity of insulin or 32,33 split proinsulin in the intact proinsulin assay. Insulin did not significantly cross-react in the assay for 32, 33 split proinsulin and the cross-reactivity of intact proinsulin in this assay was $84 \%$. Assay values of 32,33 split proinsulin were corrected for this by subtracting the corresponding proinsulin cross-reactivity. The assay of 32,33 split proinsulin cross-reacts equally with 32,33 , des-32 and des-31, 32 split proinsulin. We use the term 
Table 2. Baseline IRI, specific insulin, intact proinsulin, and 32, 33 split proinsulin concentrations, and proinsulin: specific insulin ratios

\begin{tabular}{|c|c|c|c|c|c|c|}
\hline & \multicolumn{3}{|l|}{ Subjects } & \multicolumn{3}{|l|}{$P$-values } \\
\hline & $\begin{array}{l}\text { Cases } \\
(n=69)\end{array}$ & $\begin{array}{l}\text { Random } \\
\text { controls } \\
(n=69)\end{array}$ & $\begin{array}{l}\text { Matched } \\
\text { controls } \\
(n=69)\end{array}$ & Overall & $\begin{array}{l}\text { Cases vs } \\
\text { random } \\
\text { controls }\end{array}$ & $\begin{array}{l}\text { Cases vs } \\
\text { matched } \\
\text { controls }\end{array}$ \\
\hline Fasting IRI (pmol/l) & $\begin{array}{l}93 \\
(70,114)\end{array}$ & $\begin{array}{l}78^{a} \\
(57,115)\end{array}$ & $\begin{array}{l}91 \\
(72,122)\end{array}$ & 0.013 & 0.019 & 0.874 \\
\hline $\begin{array}{l}\text { Fasting specific } \\
\text { insulin }(\mathrm{pmol} / \mathrm{1})\end{array}$ & $\begin{array}{l}123 \\
(77,154)\end{array}$ & $\begin{array}{l}108 \\
(74,143)\end{array}$ & $\begin{array}{l}118 \\
(83,145)\end{array}$ & 0.271 & 0.202 & 0.826 \\
\hline $\begin{array}{l}\text { Fasting intact } \\
\text { proinsulin (pmol/l) }\end{array}$ & $\begin{array}{l}7.3 \\
(4.8,10.0)\end{array}$ & $\begin{array}{l}5.7^{\mathrm{b}} \\
(3.8,9.0)\end{array}$ & $\begin{array}{l}5.9^{\mathrm{a}} \\
(4.0,7.7)\end{array}$ & 0.011 & 0.005 & 0.019 \\
\hline $\begin{array}{l}\text { Fasting } 32,33 \\
\text { split proinsulin }(\mathrm{pmol} / \mathrm{l})\end{array}$ & $\begin{array}{l}10.4 \\
(7.1,18.0)\end{array}$ & $\begin{array}{l}7.3^{\mathrm{b}} \\
(4.5,13.0)\end{array}$ & $\begin{array}{l}9.5 \\
(6.6,13.5)\end{array}$ & 0.004 & 0.002 & 0.530 \\
\hline $\begin{array}{l}\text { Sum of insulin-like } \\
\text { molecules }(\mathrm{pmol} / 1)\end{array}$ & $\begin{array}{l}136 \\
(93,171)\end{array}$ & $\begin{array}{l}121 \\
(81,165)\end{array}$ & $\begin{array}{l}132 \\
(93,166)\end{array}$ & 0.163 & 0.090 & 0.915 \\
\hline
\end{tabular}

Random control subjects matched for gender only

Matched control subjects matched for gender, glucose tolerance status (normal or impaired glucose tolerance), and BMI $p$-value for ANOVA based on the natural log transformation of the dependent variables.
${ }^{\mathrm{a}} p<0.05,{ }^{\mathrm{b}} p<0.01$

Data are median, 25 th and 75 th percentiles
32,33 split proinsulin to indicate the sum of these three molecules and we believe that the majority of these molecules are probably des-31, 32 split proinsulin. The sensitivity limit of the intact proinsulin and 32,33 split proinsulin assays was $1.25 \mathrm{pmol} / \mathrm{l}$. One subject had an intact proinsulin level and three subjects had 32,33 split proinsulin levels below the sensitivity limits.

In the Tables the sum of the insulin-like molecules denotes the sum of fasting specific insulin, intact proinsulin and 32, 33 split proinsulin concentrations. The sum of the insulin-like molecules exceeds the measure of fasting IRI concentration by about $40 \%$. A possible explanation for this is the difference in calibration, since fasting IRI was determined at the laboratory of the Department of Internal Medicine at the University of Kuopio, Finland and specific insulin, intact proinsulin and 32, 33 split proinsulin were determined at the laboratory of the Department of Clinical Biochemistry at Addenbrook's Hospital, Cambridge, UK.

\section{Statistical analysis}

Data analyses were performed with the SPSS/PC + and SAS statistical software. The results for continuous variables are given as mean \pm SD or median and 25 th and 75 th percentiles (skewed variables). The chi-squared test $\left(\chi^{2}\right)$ was used for evaluating differences in proportions between groups. Analysis of variance (ANOVA) was used to compare the means between groups. Due to multiple comparisons in Tables 1 and 2, a significance level of $0.025(0.05 / 2=0.025$; significance level divided by the number of comparisons) should be used for testing pairwise contrasts to keep the overall experimental error rate at 0.05 . The risk of developing diabetes associated with baseline specific insulin and proinsulin levels and ratios was assessed by proportional hazards regression analyses and calcu- lating standardized odds ratios (OR) (relative odds associated with a difference of 1 SD in baseline insulin or proinsulin level or ratio). To improve skewness and kurtosis of the distributions, IRI, specific insulin, intact proinsulin, and 32, 33 split proinsulin values and proinsulin: specific insulin and split proinsulin: specific insulin ratios were natural log-transformed for statistical analyses and then back-transformed to their natural units for presentation in the Tables.

\section{Results}

Baseline characteristics of the study population are shown in Table 1. There were no differences in age or waist-to-hip ratio between prediabetic subjects and random or matched control groups. The proportion of men was identical in all three groups since they were matched for gender. Random control subjects had lower prevalence of impaired glucose tolerance, BMI, and fasting and 2-h plasma glucose concentrations compared to prediabetic subjects. There were no differences in the proportion of subjects with impaired glucose tolerance, BMI, or 2-h plasma glucose concentrations between prediabetic and matched control subjects. However, matched control subjects had slightly but significantly lower $(0.2 \mathrm{mmol} / \mathrm{l})$ fasting plasma glucose levels compared to prediabetic subjects.

Table 2 shows baseline fasting IRI, specific insulin, intact proinsulin, and 32, 33 split proinsulin concentrations, sum of insulin-like molecules, and proinsulin: specific insulin ratios. Random control subjects 
Table 3. Standardized odds ratios for developing NIDDM by proportional hazards regression analyses

\begin{tabular}{|c|c|c|c|c|c|c|}
\hline & \multicolumn{3}{|c|}{ Cases vs random control subjects } & \multicolumn{3}{|c|}{ Cases vs matched control subjects } \\
\hline & OR & $95 \% \mathrm{Cl}$ & $p$-value & OR & $95 \% \mathrm{Cl}$ & $p$-value \\
\hline Age & 0.81 & $0.54-1.22$ & 0.316 & 0.92 & $0.67-1.26$ & 0.605 \\
\hline Impaired glucose tolerance & $13.0^{c}$ & $4.02-42.1$ & $<0.001$ & - & - & - \\
\hline BMI & $1.74^{\mathrm{b}}$ & $1.23-2.45$ & 0.002 & - & - & - \\
\hline Waist: hip ratio & 20.7 & $0.48-892$ & 0.114 & - & - & - \\
\hline Fasting intact proinsulin & $1.39^{\mathrm{a}}$ & $1.06-1.82$ & 0.016 & $1.34^{\mathrm{a}}$ & $1.04-1.73$ & 0.024 \\
\hline Fasting 32,33 split proinsulin & $1.51^{b}$ & $1.11-2.06$ & 0.009 & 1.12 & $0.83-1.51$ & 0.458 \\
\hline Sum of insulin-like molecules & 1.23 & $0.94-1.60$ & 0.127 & 1.02 & $0.71-1.47$ & 0.915 \\
\hline Proinsulin: specific insulin ratio & 1.28 & $0.98-1.67$ & 0.071 & $1.37^{\mathrm{a}}$ & $1.05-1.79$ & 0.020 \\
\hline Split proinsulin: specific insulin ratio & $1.36^{\mathrm{b}}$ & $1.05-1.76$ & 0.019 & 1.15 & $0.89-1.49$ & 0.289 \\
\hline
\end{tabular}

${ }^{\mathrm{a}} p<0.05 ;{ }^{\mathrm{b}} p<0.01 ;{ }^{\mathrm{c}} p<0.001$

had significantly lower fasting IRI levels compared to prediabetic subjects. However, matched control and prediabetic subjects did not differ regarding fasting IRI concentrations. Random control subjects had somewhat lower fasting specific insulin concentrations compared to prediabetic subjects, but this difference was not statistically significant. There was no difference in fasting serum specific insulin concentration between prediabetic and matched control subjects. Random control subjects had significantly lower intact proinsulin and 32, 33 split proinsulin concentrations and lower split proinsulin: insulin ratio at baseline compared to prediabetic subjects. Furthermore, the proinsulin: insulin ratio was lower in random control subjects compared to prediabetic subjects, but this difference was not quite statistically significant. Matched control subjects had significantly lower intact proinsulin concentrations and proinsulin: insulin ratios at baseline compared to prediabetic subjects. In contrast, 32, 33 split proinsulin concentration and split proinsulin: insulin ratio were not different between matched control and prediabetic subjects. The sum of insulin-like molecules was somewhat lower in random control subjects compared to prediabetic subjects, but this difference was not statistically significant. There was no difference in the sum of insulin-like molecules between prediabetic and matched control subjects.

The risk of developing diabetes associated with baseline fasting IRI, specific insulin, intact proinsulin, and 32, 33 split proinsulin concentrations, the sum of insulin-like molecules, and proinsulin: specific insulin and split proinsulin: specific insulin ratios was also assessed by proportional hazards regression analyses and calculating standardized odds ratios (Table 3). For comparison we also estimated the risk of developing diabetes related to baseline age, fasting glucose level, impaired glucose tolerance, BMI, and waist-to-hip ratio. When prediabetic subjects were compared to the random control subjects, a
$1 \mathrm{SD}$ increase in baseline fasting IRI concentration was associated with a 1.34-fold risk of developing diabetes $(p=0.046)$. However, the fasting specific insulin concentration was not significantly related to the risk of developing diabetes $(p=0.238)$. On the other hand, a $1 \mathrm{SD}$ increase in baseline intact proinsulin concentration was associated with a 1.39 -fold risk $(p=0.016)$ of developing diabetes. A $1 \mathrm{SD}$ increase in 32, 33 split proinsulin concentration was associated with a 1.51-fold risk ( $p=0.009)$ of developing diabetes. Split proinsulin: insulin ratio was also associated with a significantly increased risk of diabetes $(\mathrm{OR}=1.36, p=0.019)$. Proinsulin: insulin ratio was related to a 1.28-fold risk of developing diabetes, but this association was not quite statistically significant $(p=0.071)$. The sum of insulin-like molecules was not significantly related to the risk of diabetes $(p=0.127)$. Impaired glucose tolerance was clearly the strongest risk factor for diabetes $(\mathrm{OR}=13.0$, $p<0.001)$. A 1 SD increase in baseline fasting glucose concentration was associated with a 1.78 -fold risk $(p<0.001)$ of developing diabetes when prediabetic subjects were compared to random control subjects. The risk of diabetes related to baseline BMI ( $\mathrm{OR}=1.74, p=0.002$ ) was of the same magnitude as the risk related to baseline fasting glucose. Age and waist-to-hip ratio were not significantly related to the risk of developing diabetes.

When prediabetic subjects were compared to matched control subjects (Table 3 ), baseline fasting IRI $(p=0.858)$ or fasting specific insulin $(p=0.820)$ concentrations were not significantly related to the risk of developing diabetes. In contrast, a 1 SD increase in baseline intact proinsulin concentration ( $O R=$ $1.34, p=0.024$ ) and proinsulin: specific insulin ratio (OR $=1.37, p=0.020)$ was significantly associated with the risk of diabetes. The 32,33 split proinsulin $(p=0.458)$ or split proinsulin: insulin ratios $(p=0.289)$ were not associated with the risk of developing diabetes when cases were compared to mat- 
Table 4. Fasting glucose and BMI adjusted standardized odds ratios for developing NIDDM by proportional hazards regression analyses

\begin{tabular}{llcl}
\hline & \multicolumn{2}{l}{ Cases vs random control subjects } & \\
\cline { 3 - 3 } & & $95 \% \mathrm{Cl}$ & $p$-value \\
\hline Fasting IRI & 0.98 & $0.62-1.54$ & 0.930 \\
Fasting specific insulin & 0.84 & $0.59-1.19$ & 0.329 \\
Fasting intact proinsulin & 1.07 & $0.78-1.47$ & 0.678 \\
Fasting 32, 33 split proinsulin & 1.32 & $0.90-1.93$ & 0.151 \\
Sum of insulin-like molecules & 0.96 & $0.70-1.32$ & 0.802 \\
Proinsulin: specific insulin ratio & 1.22 & $0.90-1.64$ & 0.191 \\
Split proinsulin: specific insulin ratio & $1.54^{\mathrm{a}}$ & $1.08-2.19$ & 0.017 \\
\hline
\end{tabular}

${ }^{\mathrm{a}} p<0.05$

ched control subjects. Neither was the sum of insulinlike molecules related to the risk of diabetes $(p=0.915)$. A 1 SD increase in fasting glucose concentration was associated with a 1.50 -fold risk of developing diabetes when prediabetic subjects were compared to matched controll subjects.

Although matched control and prediabetic subjects were matched for glucose tolerance status, prediabetic subjects had significantly higher fasting glucose concentrations compared to matched control subjects. Numerous follow-up studies have shown that an elevated glucose level is a strong predictor of diabetes development. Therefore, we also performed proportional hazards regression analyses comparing prediabetic subjects to random control subjects adjusting for baseline fasting glucose concentration and BMI (Table 4). Baseline split proinsulin: specific insulin ratio was significantly related to the risk of diabetes even after adjustment for fasting glucose and BMI. However, the association of fasting IRI, intact proinsulin and 32,33 split proinsulin concentrations with the development of diabetes was not independent of fasting glucose level and BMI. Furthermore, we performed proportional hazards regression analyses comparing prediabetic subjects to matched control subjects controlling for baseline fasting glucose concentration (data not shown). Baseline intact proinsulin $[\mathrm{OR}=1.30,95 \%$ confidence interval $(\mathrm{CI})=$ $1.00-1.69, p=0.048]$ and proinsulin: specific insulin ratio $(\mathrm{OR}=1.38,95 \% \mathrm{CI}=1.05-1.82, p=0.021)$ were significantly associated with the risk of developing diabetes even after adjusting for fasting glucose level. In fact, after adjustment for fasting glucose, standardized odds ratios were not markedly different from the unadjusted odds ratios (Table 3 ).

\section{Discussion}

Impaired insulin secretion plays an essential role in the pathogenesis of NIDDM. There is extensive evidence that in populations at high risk of diabetes, the primary defect in the development of NIDDM is insulin resistance $[6,8]$ with consequent hyperinsulin- aemia [1-4]. According to studies in non-diabetic relatives of NIDDM subjects the defect in insulin resistance is primarily in non-oxidative glucose metabolism $[5,29]$. Nevertheless, in addition to insulin resistance, concomitant impairment of insulin secretion is thought to be necessary for progression to overt diabetes [7]. Accordingly, among subjects with impaired glucose tolerance a defect in insulin secretion which was manifested as low 2 -h $\mathrm{h}$ insulin levels after an oral glucose load predicted conversion to NIDDM $[2,30]$. Furthermore, several studies have suggested that in some white populations impaired insulin secretion may be the primary defect in the pathogenesis of NIDDM [9-11].

Our study is the first prospective study to show that beta-cell dysfunction, manifested as disproportionately increased proinsulin relative to insulin levels, is associated with short-term conversions to diabetes in elderly subjects. Subjects who subsequently developed diabetes in the present study were more obese compared to random control subjects which could explain their higher absolute intact proinsulin and 32,33 split proinsulin concentrations as shown in previous studies $[15,22,31]$. However, prediabetic subjects also had significantly increased split proinsulin: insulin ratios compared to random control subjects implying that the elevation in split proinsulin levels was disproportionate relative to insulin levels. Furthermore, the proinsulin: insulin ratio was higher in the prediabetic subjects compared to random control subjects, although this difference was not statistically significant. There is evidence that proinsulin levels are not disproportionately increased relative to insulin levels in obesity $[15,22,31]$ and therefore obesity cannot explain the elevated split proinsulin: intact insulin ratio in prediabetic subjects compared to random control subjects.

Absolute proinsulin levels are also elevated in subjects with impaired glucose tolerance [15, 18-22]. Furthermore, in several studies elevation of fasting proinsulin concentrations was disproportionate relative to insulin concentrations in subjects with impaired glucose tolerance $[19,21,22]$. Thus, it is possible that the high prevalence of impaired glucose tol- 
erance in prediabetic subjects could explain their elevated intact proinsulin and 32,33 split proinsulin concentrations and proinsulin: specific insulin and split proinsulin: specific insulin ratios compared to random control subjects. Accordingly, intact proinsulin and 32,33 split proinsulin concentrations were not significantly related to the risk of diabetes after adjusting for fasting glucose level and BMI (Table 4). However, the association of split proinsulin: specific insulin ratio with the risk of developing diabetes was independent of baseline fasting glucose level and BMI (Table 4). Furthermore, we also found in this study that prediabetic subjects had elevated intact proinsulin concentrations and proinsulin: insulin ratio compard to control subjects matched for glucose tolerance status and BMI. Moreover, the increased risk of developing diabetes associated with baseline intact proinsulin concentration and proinsulin: specific insulin ratio was independent of baseline fasting glucose concentration in prediabetic subjects compared to matched control subjects (data not shown). This means that increased proinsulin: insulin ratio in prediabetic subjects was not entirely due to the high prevalence of impaired glucose tolerance or somewhat higher fasting glucose concentration compared to matched control subjects but was related to the prediabetic state.

It is unclear why the risk of diabetes was more closely related to the split proinsulin: insulin ratio if prediabetic subjects were compared to random control subjects and to proinsulin: insulin ratio if prediabetic subjects were compared to matched control subjects. A possible explanation is that the 32,33 split proinsulin assay is less precise at low serum concentrations than the assay of intact proinsulin. This is due to the 32,33 split proinsulin assay being inherently a little less sensitive than the assay of intact proinsulin and the need to subtract the intact proinsulin cross-reactivity which adds further potential error.

Impaired glucose tolerance was clearly the strongest risk factor for diabetes in the present study (Table 3). Fasting glucose level and BMI at baseline were also strong predictors of the development of diabetes which is in accordance with previous findings $[3,4]$. However, baseline proinsulin concentration and proinsulin: specific insulin ratio were essentially as valid as predictors of diabetes risk as was fasting glucose when prediabetic subjects were compared to subjects matched for obesity and impaired glucose tolerance.

We have previously reported [25] that a high fasting IRI level is a risk factor for developing diabetes in the same elderly cohort as this case-control study. That was also the situation in the present case-control study when subjects who subsequently developed diabetes were compared to random control subjects (Tables 2,3). Although prediabetic subjects also had somewhat higher fasting specific insulin concentra- tions than random control subjects, specific insulin concentration was not significantly associated with the risk of developing diabetes. There were no differences in the fasting IRI or specific insulin levels between prediabetic subjects and matched control subjects implying that elevated insulin levels in prediabetic subjects were at least partially related to impaired glucose tolerance and obesity. Accordingly, the association of baseline fasting IRI concentration with the risk of developing diabetes was not independent of fasting glucose level and BMI when prediabetic subjects were compared to random control subjects (Table 4). This contrasts with earlier studies in which fasting IRI concentration predicted the development of diabetes even after adjustment for obesity [6] or fasting glucose level and obesity [3]. These divergent results may be due to the lower power of the nested case-control design of the present study as compared to prospective cohort design of the previous studies $[3,6]$. Furthermore, our study population was elderly and the follow-up period was only 3.5 years; therefore the lack of association between fasting IRI and specific insulin and subsequent diabetes when prediabetic subjects were compared to matched control subjects in this study may not be generalizable to younger age groups or to longer periods of follow-up. Lastly, we used specific insulin as contrasted to IRI in previous studies. In Tables 2 and 3 fasting IRI was significantly related to risk of diabetes when cases were compared to random control subjects.

Disproportionately high fasting proinsulin relative to insulin levels in prediabetic subjects could be caused by increased clearance of insulin or decreased clearance of proinsulin. Although the metabolic clearance rate of proinsulin is markedly lower compared to insulin [32], in the basal state proinsulin and insulin concentrations are expected to be in equilibrium. Therefore, we only assessed the fasting proinsulin: insulin ratio. The disproportionately elevated fasting proinsulin concentration could also be due to hypersecretion of proinsulin by secretion of immature proinsulin-rich granules in beta cells in response to an increased demand for insulin [18]. The prediabetic subjects were, however, matched with a control group for obesity and impaired glucose tolerance which are both conditions associated with an increased demand for insulin. It is possible that prediabetic subjects had a reduced beta-cell mass which would require increased insulin production of each beta cell to maintain the same degree of insulin output. Finally, hyperproinsulinaemia in prediabetic subjects could be related to an intrinsic beta-cell defect and impaired processing of proinsulin or release of insulin [12].

In conclusion, our results show that prediabetic subjects have disproportionately increased proinsulin relative to insulin levels but similar specific insulin levels compared to matched control subjects. Thus, in elderly prediabetic subjects disproportion- 
ately increased proinsulin levels, an indicator of defective insulin secretion, was associated with conversion to diabetes over a short period of time.

Acknowledgements. This work was supported by grants from the Medical Research Council of the Academy of Finland.

\section{References}

1. Saad MF, Knowler WC, Pettitt DJ, Nelson RG, Mott DM, Bennett PH (1989) Sequential changes in serum insulin concentration during development of non-insulin-dependent diabetes. Lancet I: 1356-1359

2. Sicree RA, Zimmet PZ, King HOM, Coventry JS (1987) Plasma insulin response among Nauruans. Prediction of deterioration in glucose tolerance over 6 yr. Diabetes 36: 179_ 186

3. Haffner SM, Stern MP, Mitchell BD, Hazuda HP, Patterson JK (1990) Incidence of type II diabetes in Mexican Americans predicted by fasting insulin and glucose levels, obesity, and body-fat distribution. Diabetes 39: 283-288

4. Charles MA, Fontbonne A, Thibult N, Warnet J-M, Rosselin GE, Eschwege E (1991) Risk factors for NIDDM in white population: Paris Prospective Study. Diabetes 40: 796-799

5. Eriksson J, Franssila-Kallunki A, Ekstrand A, Saloranta C, Widen E, Schalin C, Groop L (1989) Early metabolic defects in persons at increased risk for non-insulin-dependent diabetes mellitus. N Engl J Med 321: 337-343

6. Warram JH, Martin BC, Krolewski AS, Soeldner JS, Kahn CR (1990) Slow glucose removal rate and hyperinsulinemia precede the development of type II diabetes in the offspring of diabetic parents. Ann Intern Med 113: 909-915

7. Saad MF, Knowler WC, Pettitt DJ, Nelson RG, Charles MA, Bennett PH (1991) A two-step model for development of non-insulin-dependent diabetes. Am J Med 90: 229-235

8. Lillioja S, Mott DM, Spraul M et al. (1993) Insulin resistance and insulin secretory dysfunction as precursors of non-insulin-dependent diabetes mellitus. Prospective studies of Pima Indians. N Engl J Med 329: 1988-1992

9. Luft R, Wajngot A, Efendic S (1981) On the pathogenesis of maturity-onset diabetes. Diabetes Care 4: 58-63

10. O'Rahilly SP, Nugent Z, Rudenski AS, Hosker JP, Burnett MA, Darling P, Turner RC (1986) Beta-cell dysfunction, rather than insulin insensitivity, is the primary defect in familial type 2 Diabetes. Lancet II: 360-364

11. Arner P, Pollare T, Lithell H (1991) Different aetiologies of type 2 (non-insulin-dependent) diabetes mellitus in obese and non-obese subjects. Diabetologia 34: 483-487

12. Porte D, Kahn SE (1989) Hyperproinsulinemia and amyloid in NIDDM. Clues to etiology of islet $\beta$-cell dysfunction? Diabetes 38: 1333-1336

13. Ward WK, LaCava EC, Paquette TL, Beard JC, Wallum BJ, Porte Jr. D (1987) Disproportionate elevation of immunoreactive proinsulin in type 2 (non-insulin-dependent) diabetes mellitus and in experimental insulin-resistance. Diabetologia 30: 698-702

14. Temple RC, Carrington CA, Luzio SD, Owens DR, Schneider AE, Sobey WJ, Hales CN (1989) Insulin deficiency in non-insulin-dependent diabetes. Lancet I: 293-295

15. Saad MF, Kahn SE, Nelson RG et al. (1990) Disproportionately elevated proinsulin in Pima Indians with noninsulindependent diabetes mellitus. J Clin Endocrinol Metab 70: $1247-1253$
16. Clark PM, Levy JC, Cox L, Burnett M, Turner RC, Hales CN (1992) Immunoradiometric assay of insulin, intact proinsulin and 32, 33 split proinsulin and radioimmunoassay of insulin in diet-treated type 2 (non-insulin-dependent) diabetic subjects. Diabetologia 35: 469-474

17. Davis SN, Piatti PM, Monti L, Brown MD, Branch W, Hales CN, Alberti KGMM (1993) Proinsulin and insulin concentrations following intravenous glucose challenge in normal, obese, and non-insulin-dependent diabetic subjects. Metabolism 42: 30-35

18. Yoshioka N, Kuzuya T, Matsuda A, Taniguchi M, Iwamoto $Y$ (1988) Serum proinsulin levels at fasting and after oral glucose load in patients with type 2 (non-insulin-dependent) diabetes mellitus. Diabetologia 31: $355-360$

19. Williams DRR, Byrne C, Clark PMS (1991) Raised proinsulin concentration as early indicator of $\beta$-cell dysfunction. BMI 303: 95-96

20. Reaven GM, Chen Y-DI, Hollenbeck CB, Sheu WHH, Ostrega D, Polonsky KS (1993) Plasma insulin, C-peptide, and proinsulin concentrations in obese and nonobese individuals with varying degrees of glucose tolerance. $\mathrm{J}$ Clin Endocrinol Metab 76: 44-48

21. Davies MJ, Rayman G, Gray IP, Day IL, Hales CN (1993) Insulin deficiency and increased plasma concentration of intact and $32 / 33$ split proinsulin in subjects with impaired glucose tolerance. Diabet Med 10: 313-320

22. Shiraishi I, Iwamoto Y, Kuzuya T, Matsuda A, Kumakura S (1991) Hyperinsulinaemia in obesity is not accompanied by an increase in serum proinsulin/insulin ratio in groups of human subjects with and without glucose intolerance. Diabetologia 34: 737-741

23. Mykkänen L, Laakso M, Uusitupa M, Pyörälä K (1990) Prevalence of diabetes and impaired glucose tolerance in elderly subjects and their association with obesity and family history of diabetes. Diabetes Care 13: 1099-1105

24. Mykkänen L, Laakso M, Penttilä I, Pyörälä K (1991) Asymptomatic hyperglycemia and cardiovascular risk factors in the elderly. Atherosclerosis 88: 153-161

25. Mykkänen L, Kuusisto J, Pyörälä K, Laakso M (1993) Cardiovascular disease risk factors as predictors of type 2 (non-insulin-dependent) diabetes mellitus in elderly subjects. Diabetologia 36: 553-559

26. World Health Organization (1985) Diabetes mellitus. Report of a WHO Study Group. (Technical Report Series No. 727). World Health Organization, Geneva

27. Hales CN, Randle PJ (1963) Immunoassay of insulin with insulin antibody precipitate. Biochem J 88: 137-146

28. Sobey WJ, Beer SF, Carrington CA et al. (1989) Sensitive and specific two-site immunoradiometric assays for human insulin, proinsulin, 65-66 split and 32-33 split proinsulins. Biochem J 260: 535-541

29. Gulli G, Ferrannini E, Stern M, Haffner S, DeFronzo RA (1992) The metabolic profile of NIDDM is fully established in glucose-tolerant offspring of two Mexican-American NIDDM parents. Diabetes 41: 1575--1586

30. Saad MF, Knowler WC, Pettitt DJ, Nelson RG, Mott DM, Bennett PH (1988) The natural history of impaired glucose tolerance in the Pima Indians. $N$ Engl $J$ Med 319: 1500-1506

31. Koivisto VA, Yki-Järvinen H, Hartling SG, Pelkonen R (1986) The effect of exogenous hyperinsulinemia on proinsulin secretion in normal man, obese subjects, and patients with insulinoma. J Clin Endocrinol Metab 63: 1117-1120

32. Bergenstal RM, Cohen RM, Lever E et al. (1984) The metabolic effects of biosynthetic human proinsulin in individuals with type 1 diabetes. J Clin Endocrinol Metab 58: 973979 